\title{
Overexpression of miR-221-3p affects cell proliferation, apoptosis and inflammation by targeting toll-like receptor 4 in propofol-induced rat astrocytes
}

\author{
Shuang Qi, Zinan Li, Shanshan Yu
}

Department of Anesthesiology, China-Japan Union Hospital of Jilin University, Changchun City, Jilin Province, P.R. China

Submitted: 1 March 2021; Accepted: 21 July 2021

Online publication: 13 August 2021

Arch Med Sci

DOI: https://doi.org/10.5114/aoms/140396

Copyright $\odot 2022$ Termedia \& Banach

\begin{abstract}
Introduction: Growing evidence indicates that propofol has neurotoxic effects on the brains of developing rodents, leading to neuronal cell death, neurodegeneration, and brain injury. Also, microarray data indicated that the miR-221-3p in mRNA and protein expression levels were negatively altered in propofol-treated rat astrocytes; however, the effect of miR-221-3p on propofol-treated astrocytes remains unclear. Thus, we aimed to evaluate the function and mechanism of miR-221-3p in propofoltreated astrocytes.

Material and methods: Ectopic miR-221-3p was transfected into rat astrocytes, and the Cell Counting Kit-8 assay and flow cytometry were performed to evaluate cell growth and apoptosis. The mRNA levels of toll-like receptors 4 (TLR4), nuclear factor kappa B, interleukin-6, interleukin-13, myeloid differentiation primary response 88 (MyD88), caspase-3, caspase-12, STAT3, and GRP78 were detected using quantitative real-time polymerase chain reaction. The proteins of TLR4 and MyD88 were determined using Western blotting. The association between miR-221-3p and TLR4 was measured using the Dual-Luciferase Reporter Assay (Promega Corporation, Wisconsin, USA). Then, siTLR4 was transfected with 293 T cells to study the role of TLR4 in astrocytes with propofol treatment.

Results: The miR-221-3p expression in rat astrocytes was markedly suppressed by propofol treatment. The miR-221-3p mimic transfection in propofoltreated astrocytes effectively reduced the suppressive effect of propofol on astrocyte growth, repressed the propofol-induced apoptosis in rat astrocytes, and decreased the cell number during the G2/M phase. The expression of MyD88 and TLR4 was induced by propofol, whereas the transfection of miR-221-3p mimics dramatically reduced expression of these genes at the mRNA and protein expression level. After that, TLR4 was found to be the target of miR-221-3p using the Dual-Luciferase Reporter Assay. Furthermore, knockdown of TLR4 could suppress the apoptosis rate in propofol-treated astrocytes.

Conclusions: This study revealed that miR-221-3p might prevent astrocytes from propofol-induced damage by targeting TLR4.
\end{abstract}

Key words: apoptosis, cell cycle, TLR4, MyD88.

\section{Introduction}

Propofol (2,6-diisopropyl phenol) is used as a general intravenous anesthetic worldwide, due to its fast onset and low postoperative ad-

\author{
Corresponding author: \\ Shanshan Yu \\ Department of Anesthesiology \\ China-Japan Union Hospital \\ of Jilin University \\ 126 Xiantai Street \\ Changchun City, Jilin Province \\ 130033, P.R. China \\ Phone: 86-431-84995481 \\ Fax: 86-431-84995481 \\ E-mail: \\ yushanshan@jlu.edu.cn
}


verse reactions [1, 2]. Previous studies showed that propofol also was regarded as an anesthetic for children and pregnant women [3, 4]. However, in the great majority of clinical pediatric practices, propofol is considered as an off-label choice [5]. More and more studies have found that propofol might cause neurotoxicity and neurogenic damage. At present, there is a lack of evidence to support the safe use of propofol $[6,7]$. Astrocytes are the most abundant glial cells in the central nervous system [8], which provide critical support for brain development, neuronal differentiation, and neurotransmitter homeostasis in the developing and adult CNS $[9,10]$. Thus, the potential role of propofol in developing anesthesia strategies may be reconsidered.

MicroRNAs (miRNAs) are short non-coding RNAs (18-23 nt) that bind to the seed sequences within the 3'UTR region of target mRNAs to regulate translational repression or degradation of target mRNAs [11]. In addition, miRNAs dysregulation was associated with neurodegenerative disease $[10,12]$. Some miRNAs are related to inflammatory pathways, such as miR-155, miR-146a, miR-21 and miR-124 [13]. Also, studies have proven that miRNAs affect the astrocyte cell cycle and their conversion into neurons, such as miR-146a [14], miR-338 [15], and miR-143-3p [16]. In addition, previous studies indicated that miR-221-3p is related to inflammatory conditions $[17,18]$. Up to now, miR-221-3p expression has been mainly reported to occur in pathological conditions associated with cancer, such as thyroid cancer [19], non-small cell lung cancer [20] and breast cancer [21]. Moreover, Feng et al. [22] suggested that miR-221-3p could be used as a biomarker for mediated activation in astrocytes. Toll-like receptors (TLRs) are a family of transmembrane pattern recognition receptors that are mainly expressed on immune cells [23]. TLRs could induce a series of cell signal transduction pathways, release inflammatory mediators, and activate the adaptive immune system [24]. In addition, previous studies have indicated that miR-221-3p could medicate the inflammatory response in human umbilical vein endothelial cells through the target gene TLR4 [17].

However, few studies have focused on the function of miR-221-3p on propofol-treated astrocytes through TLR4. Thus, in our study, we explored the function of miR-221-3p in astrocytes treated with propofol. First, the Cell Counting Kit- 8 assay and flow cytometry were carried out to assess the astrocyte cell growth and apoptosis after transfecting miR-221-3p. After that, siTLR4 was transfected with 293T cells to study the role of TLR4 in astrocytes with propofol treatment.

\section{Material and methods}

\section{Animals}

Pregnant Sprague-Dawley (SD) rats were obtained from Shanghai Laboratory Animal Center (Shanghai, China). The rats were raised using adequate food and water under constant temperature $\left(23 \pm 1^{\circ} \mathrm{C}\right)$ and maintained on an adequate light-dark cycle for $12 \mathrm{~h}$.

\section{Cell cultures}

Twenty-four hours after birth, newborns were euthanized. The mice were disinfected with $75 \%$ alcohol. We removed the cerebral cortex from the skull and carefully dissected the meninges. Moreover, we cut the brain tissues into small pieces, which were digested using $4 \mathrm{ml}$ of Hanks' Balanced Salt Solution (HBSS) (Thermo Fisher Scientific, MA, USA). Then, $1 \mathrm{ml}$ of DNase, $4 \mathrm{ml}$ of HBSS, and $1 \mathrm{ml}$ of trypsin (2.5\%) were added to a $10 \mathrm{ml}$ volume and the specimens underwent a water bath at $37^{\circ} \mathrm{C}$ for $15 \mathrm{~min}$.

Isolated cells were transferred into a fresh $50 \mathrm{ml}$ tube and were washed using modified Eagle's medium (MEM) (Whitaker Bioproduct, MD, USA) with $10 \%$ characterized horse serum (Hyclone, UT, USA) to stop trypsin activity. Dissociated cells were collected through centrifugation at $1000 \mathrm{rpm}$ for $5 \mathrm{~min}$. Then, the MEM containing $10 \%$ horse serum was added and re-suspended. 293T cells were obtained from the Cell Bank of Shanghai Academy of Health Sciences (Shanghai, China), cultured in Gibco Dulbecco's Modified Eagle Medium (DMEM) (ME100202P1; Thermo Fisher Scientific, MA, USA), and added to $10 \%$ fetal bovine serum (FBS) (10091-148; Thermo Fisher Scientific, MA, USA), which were incubated in $5 \%$ $\mathrm{CO}_{2}$ at $37^{\circ} \mathrm{C}$.

\section{Identification of astrocytes}

The glial fibrillary acidic protein (GFAP) expression in astrocytes was identified using immunofluorescence staining. In short, we cultured the cells at a density of $4 \times 10^{5}$ cells $/ \mathrm{ml}$ in poly-l-lysinecoated coverslips. After attaching the cells to the flask, immunofluorescence staining was applied to identify astrocytes. Fresh 4\% paraformaldehyde (Solarbio, Beijing, China) was prepared for fixing the cells at $4^{\circ} \mathrm{C}$ for $30 \mathrm{~min}$. After that, we washed the cells once using phosphate-buffered saline (PBS) containing 5\% penicillin/streptomycin (Corning Inc., Corning, NY, USA) and the cells were lysed using $0.1 \%$ Triton X-100 (Solarbio, Beijing, China) at room temperature for $20 \mathrm{~min}$. Then, we washed the cells with PBS three times, and blocked using 3\% BSA in PBS for $1 \mathrm{~h}$, and combined with the primary antibody against GFAP 
(rabbit anti-GFAP, 1:800; ab7260, Abcam, Cambridge, UK). The cells were incubated overnight in a humid room at $4^{\circ} \mathrm{C}$. The cells were washed with PBS three times, and then were incubated with donkey anti-rabbit IgG-488 (Sigma-Aldrich, MO, USA) at $37^{\circ} \mathrm{C}$ for $1.5 \mathrm{~h}$ in the dark and stained with 4 0,6-diamidino-2-phenylindole (Cell Signaling Technology, Danfoss, Massachusetts, USA). All images were obtained through a fluorescence microscope (Optika, Ponteranica, Bergamo, Italy) with appropriate filters.

\section{Cell processing}

Astrocytes were treated with $10 \mu \mathrm{M}$ propofol (the propofol-treated group), and astrocytes not treated with isopropanol were used as controls (the control group). After $48 \mathrm{~h}$, we discarded the culture medium, then added $1 \mathrm{ml}$ of TRIzol (Thermo Fisher Scientific, MA, USA) to each group of cells, and stored at $-80^{\circ} \mathrm{C}$.

\section{Cell transfection}

A mimic negative control miRNA (NC mimics) and $\mathrm{miR}-221-3 p$ mimic were obtained from GenePharma (Shanghai, China). The NC mimics and miR-221-3p mimic were transfected into astrocytes by Lipofectamine 2000 Transfection Reagent (Thermo Fisher Scientific, MA, USA) based on commercial guidelines. Then, $20 \mathrm{nM}$ of miR-221$3 p$-mimics or miR-221-3p-NC was mixed with the transfection agent in Opti-MEM (Thermo Fisher Scientific, MA, USA) for $10 \mathrm{~min}$, after that added to the astrocytes seeded on 6-well plates. The cells were replaced with fresh DMEM $5 \mathrm{~h}$ after transfection and further cultured for $48 \mathrm{~h}$ until the experiments.

\section{Quantitative real-time polymerase chain reaction ( $q R T-P C R)$}

The propofol-treated and control cells were collected for total RNA extraction using commercial RNAiso Plus (Takara, Dalian, China) following the user's manual. The first-strand cDNA was reverse transcribed based on the manual of the AMV Reverse Transcriptase kit (Thermo Fisher Scientific, MA, USA). Reverse transcription of miRNAs was performed using PrimeScript RT Master Mix (Takara, Dalian, China) at $65^{\circ} \mathrm{C}$ for $5 \mathrm{~min}$. PCR reactions were conducted using SYBR Green Realtime PCR Master Mix (TOYOBO, Osaka, Japan). PCR protocols were pre-denatured at $95^{\circ} \mathrm{C}$ for $3 \mathrm{~min}$, denatured for 30 cycles at $95^{\circ} \mathrm{C}$ for $30 \mathrm{~s}$, annealed at $60^{\circ} \mathrm{C}$ for $30 \mathrm{~s}$, extended at $72^{\circ} \mathrm{C}$ for $30 \mathrm{~s}$, and finally extended at $72^{\circ} \mathrm{C}$ for $8 \mathrm{~min}$. Primers of the indicated genes were synthesized using Genscript (Nanjing, China), and the primer sequences are presented in Table I. The intracellular levels of U6 and glyceraldehyde 3-phosphate dehydrogenase (GAPDH) were used as the internal controls for miRNAs and mRNAs, respectively. The relative levels of genes and miRNAs were calculated as $2^{-\Delta \Delta C T}$ [33].

\section{Cell viability assay}

Cell viability was detected through Cell Counting Kit-8 (CCK-8) (Beyotime, Shanghai, China). Thus, the cells $(100 \mu \mathrm{l} /$ well $)$ were seeded in 96well plates for 24 h, 48 h, 72 h, and 96 h. Then, we added $10 \mu \mathrm{l}$ of CCK-8 solution to the cells and incubated in an incubator at $37^{\circ} \mathrm{C}$ for $2 \mathrm{~h}$. The absorbance at $450 \mathrm{~nm}$ (OD450) was evaluated using a microplate reader (Bio-Rad, Hercules, USA).

Table I. Primer sequences of qRT-PCR assay

\begin{tabular}{|c|c|}
\hline Primer & Sequence $\left(5^{\prime}-3^{\prime}\right)$ \\
\hline PCNA-rF & GCTTCGGCAGCACATATACTAAAAT \\
\hline PCNA-rR & CGCTTCACGAATTTGCGTGTCAT \\
\hline GFAP-rF & GGTGTGGAGTGCCTTCGTATTAG \\
\hline GFAP-rR & GGGACACTTTCAGCTCCATTTCT \\
\hline TLR4-rF & GATTGCTCAGACATGGCAGTTTC \\
\hline TLR4-rR & CACTCGAGGTAGGTGTTTCTGCTAA \\
\hline$N F-\kappa B-r F$ & GCACGGATGACAGAGGCGTGTATAAGG \\
\hline$N F-\kappa B-r R$ & GGCGGATGATCTCCTTCTCTCTGTCTG \\
\hline IL-6-rF & AAGAAAGACAAAGCCAGAGTC \\
\hline IL-6-rR & CACAAACTGATATGCTTAGGC \\
\hline IL-1 $\beta-r F$ & AGGCTGACAGACCCCAAAAGAT \\
\hline IL-1 $\beta-r R$ & CTCCACGGGCAAGACATAGGTA \\
\hline MyD88-rF & TGGTGGTTGTTTCTGACGAT \\
\hline MyD88-rR & GATCAGTCGCTTCTGTTGGA \\
\hline caspase-3-rF & GGTATTGAGACAGACAGTGG \\
\hline caspase-3-rR & CACGGGATCTGTTTCTTTGC \\
\hline caspase-12-rF & GGAAGGTAGGCAAGAGT \\
\hline caspase-12-rR & GTAGAAGTAGCGTGTCATA \\
\hline MyD88-rF & GCTGACTTGGAGCCTGATTCT \\
\hline MyD88-rR & ATGGGTGGGTGGGAGTAAA \\
\hline STAT3-rF & CACCTTGGATTGAGAGTCAAGAC \\
\hline STAT3-rR & AGGAATCGGCTATATTGCTGGT \\
\hline GRP78-rF & TCAGCCCACCGTAACAAT \\
\hline GRP78-rR & CAAACTTCTCGGCGTCAT \\
\hline $\begin{array}{l}\text { rno-miR-221- } \\
\text { 3p-RT }\end{array}$ & $\begin{array}{c}\text { GTCGTATCCAGTGCAGGGTCCGA } \\
\text { GGTATTCGCACTGGATACGACGAAACC }\end{array}$ \\
\hline $\begin{array}{l}\text { rno-miR-221- } \\
3 p-F\end{array}$ & GCGCAGCTACATTGTCTGCTG \\
\hline rno-U6-RT & CGCTTCACGAATTTGCGTGTCAT \\
\hline rno-U6-F & GCTTCGGCAGCACATATACTAAAAT \\
\hline rno-U6-R & CGCTTCACGAATTTGCGTGTCAT \\
\hline GAPDH-rF & AGACAGCCGCATCTTCTTGT \\
\hline GAPDH-rR & CTTGCCGTGGGTAGAGTCAT \\
\hline
\end{tabular}


Four groups were constructed: the propofol group, propofol + miRNA mimic group, propofol + miRNA NC group, and blank control group.

\section{Flow cytometry}

After propofol treatment for $48 \mathrm{~h}$, we collected the cells for apoptosis and cell cycle assay. The cells for apoptosis assay were first re-suspended in $1 \times$ Binding Buffer and incubated with $5 \mu \mathrm{l}$ of APC-Annexin $\mathrm{V}$ and $5 \mu \mathrm{l}$ of propidium iodide (PI) for $20 \mathrm{~min}$ in the dark at $25^{\circ} \mathrm{C}$. After gently mixing the cells with $400 \mu \mathrm{l}$ of $1 \times$ Binding Buffer, the apoptotic rate was quantified using FACSCalibur (BD Biosciences, NJ, USA) within $1 \mathrm{~h}$. In the cell cycle assay, the harvested cells were re-suspended and fixed with $5 \mathrm{ml}$ of pre-cooled $70 \%$ ethanol overnight at $4^{\circ} \mathrm{C}$. After that, we washed the cells with precooled PBS and re-suspended the cells in $0.3 \mathrm{ml}$ of PBS using a pipette gently. RNase A was added to the cells at a final concentration of 50 $\mu \mathrm{g} / \mathrm{ml}$ and digested at $37^{\circ} \mathrm{C}$ for $30 \mathrm{~min}$. The cell cycle of the cell samples was measured using flow cytometry after the final incubation with another $5 \mu \mathrm{l}$ of PI at $4^{\circ} \mathrm{C}$ for $15 \mathrm{~min}$.

\section{Western blotting}

All collected cells were washed twice with precooled PBS, lysed in a radioimmunoprecipitation assay (RIPA) buffer (Beyotime Biotechnology, Shanghai, China), then treated with ultrasound, and centrifuged at $10,000 \mathrm{~g}$ at $4^{\circ} \mathrm{C}$ for $20 \mathrm{~min}$. The protein amount was quantified using a bicinchoninic acid protein assay reagent (Thermo Fisher Scientific, MA, USA). The total protein was isolated by electrophoresis on $10 \%$ sodium dodecyl sulfate polyacrylamide gel (SDS-PAGE) and then transferred to a polyvinylidene difluoride film (Millipore, Watford, UK). The membrane was immunoblotted overnight at $4^{\circ} \mathrm{C}$ with anti-toll-like receptor 4 (TLR4) (1 : 300; Abcam, MA, USA), anti-myeloid differentiation primary response 88 (MyD88) (1: 500; Abcam, MA, USA), NF-KB (p50) (66535-1-IG, Proteintech), BAX (50599-2-IG, Proteintech), Bcl-2 (26593-1-AP, Proteintech), CASPASE3 (19677-1-AP, Proteintech), CASPASE9 (10380-1-AP, Proteintech) and anti-GAPDH (Santa Cruz Biotechnology, CA, USA). After washing in $1 \times$ TBST three times, the horseradish peroxidase-labeled secondary antibody (Jackson ImmunoResearch, PA, USA) was used for detection at room temperature for $1 \mathrm{~h}$. The protein bands were measured using an enhanced chemiluminescent system (Merck Millipore, Watford, UK). Jackson ImmunoResearch.

\section{Dual-Luciferase Reporter Assay}

We used miRwalk online software (http://zmf. umm.uni-heidelberg.de/apps/zmf/mirwalk2) to predict the target gene of miR-221-3p that can regulate TLR4.

Also, we synthesized the TLR4 3'UTR fragment containing a miR-221-3p-binding site and mutant binding site and cloned it into the pGL3 promoter plasmid (Omega, USA, D6950-02) to prepare TLR4 3'UTR-WT. Lipofectamine 2000 (11668-027; Invitrogen, Thermo Fisher Scientific, MA, USA) was used to co-transfect 293T cells with miR-221-3p or miR-NC mimics. The relative light units were detected using the Dual-Luciferase Reporter Assay System (Promega).

\section{Transient transfection with small interfering RNA (siRNA)}

The method of transfecting siRNA was according to a previous study [34]. 293T cells were transfected with siRNA-TLR4 to knock down the TLR4 gene, and the empty plasmid siNC was the control. Stable transfected 293T cells were collected using the Zeocin screening method. The mRNA expression of TLR4 was determined using qRT-PCR.

\section{Statistical analysis}

SPSS (version 21.0; IBM Corp., Armonk, NY, USA) was used to statistically process the experimental data. The quantified results are shown as mean \pm standard deviation (SD). One-way analysis of variance was used to analyze the difference between any two groups. $P<0.05$ indicated statistical significance, and $p<0.01$ indicated highly significant differences.

\section{Results}

\section{Propofol downregulated mRNA expression} of miR-221-3p expression in rat astrocytes

We isolated primary astrocytes from rat brain and ensured cell reliability using immunostaining for GFAP, a typical marker of astrocytes [25, 26]. Most cells in the microscope field displayed GFAP positivity, demonstrating that the rat astrocytes were successfully obtained (Figure 1 A). qRT-PCR results revealed that propofol treatment significantly reduced the miR-221-3p expression $(p<0.05)$ (Figure $1 \mathrm{~B}$ ).

MiR-221-3p attenuates propofol-induced decrease in cell viability in rat astrocytes

To probe the action of miR-221-3p on astrocytes during propofol treatment, miR-221-3p was ectopically imported into propofol-treated astrocytes. After transfecting astrocytes with miR-221-3p mimic and NC mimic for $48 \mathrm{~h}$, qRT-PCR results showed that miR-221-3p expression dramatically improved in the miR-221-3p mimic-transfected group as compared with the NC mimic and con- 

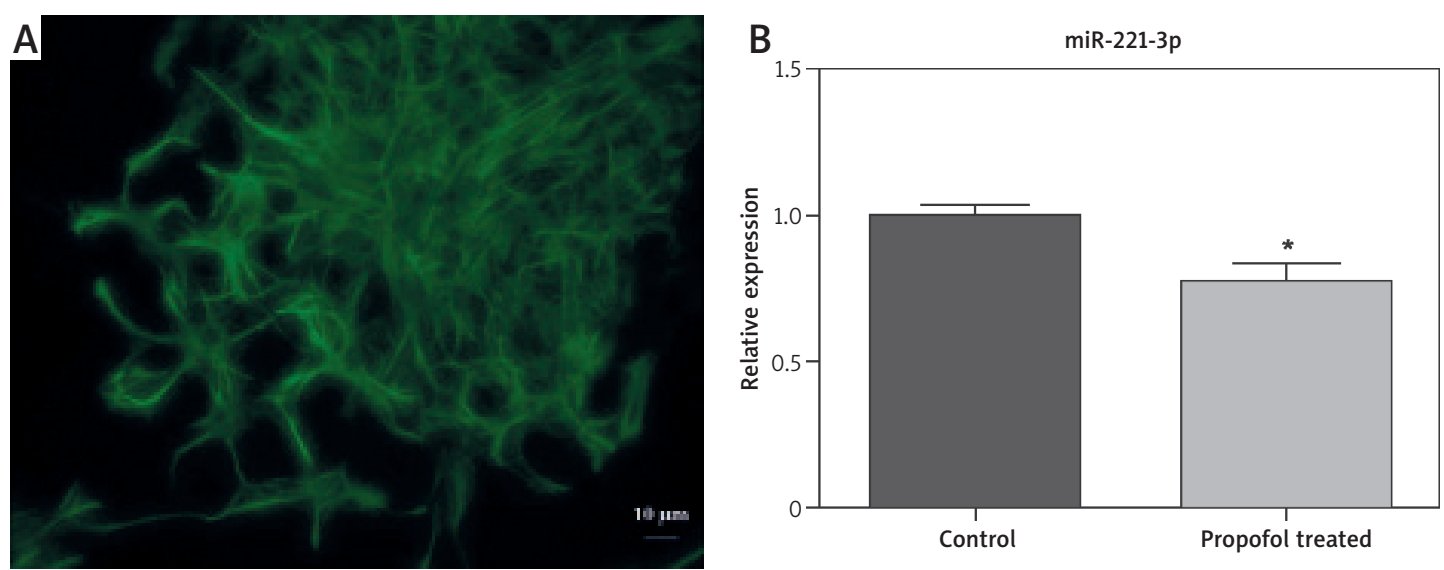

Figure 1. Propofol treatment reduces miR-221-3p expression in purified rat astrocytes. A - Purified cells were adopted for immunostaining of glial fibrillary acidic protein to verify the extraction efficiency of astrocytes. B - Isolated rat astrocytes were treated with $10 \mu \mathrm{M}$ propofol for $48 \mathrm{~h}$, miR-221-3p quantification was examined using qRT-PCR. ${ }^{*} P<0.05$ compared to the control group

trols ( $p<0.01)$, demonstrating that miR-221-3p was successfully delivered into astrocytes (Figure $2 \mathrm{~A}$ ). As shown in Figure 2 B, CCK-8 assay results indicated that the cell viability in the propofol group decreased in comparison with that in the blank group, while the cell viability in the propofol + miRNA mimic group was dramatically upregulated compared with that of the propofol group or the propofol + miRNA NC group, especially $72 \mathrm{~h}$ and 96 $\mathrm{h}$ after propofol addition $(p<0.05, p<0.01)$.

\section{MiR-221-3p regulates apoptosis and cell cycle in propofol-treated cells}

As indicated in Figure $3 \mathrm{~A}$, the apoptosis rates in the propofol + miR-221-3p mimic were decreased when compared with the propofol and propofol + miRNA NC group $(p<0.05)$, indicating that miR221-3p overexpression restored propofol-induced apoptosis in rat astrocytes. In addition, a significant increase or decrease in the proportion of cells in the G2/M phase was observed in cells transfected with miR-221-3p mimics compared with their respective NCs (Figure 3 B). Overall, these data indicated that miR-211-3p downregulation could block the cell cycle in the G2/M phase.

\section{MiR-221-3p affected propofol-induced astrocyte apoptosis}

To study the mechanism of the effect of miR221-3p on cell proliferation and apoptosis, we examined the related genes including proliferating cell nuclear antigen (PCNA), GFAP, caspase-12, nuclear factor kappa B (NF-KB), interleukin-6 (IL-6), and interleukin-1 $\beta$ (IL-1 $\beta$ ), MyD88 (myeloid differentiation factor 88), toll-like receptor 4 (TLR4), signal transducer and activator of transcription 3
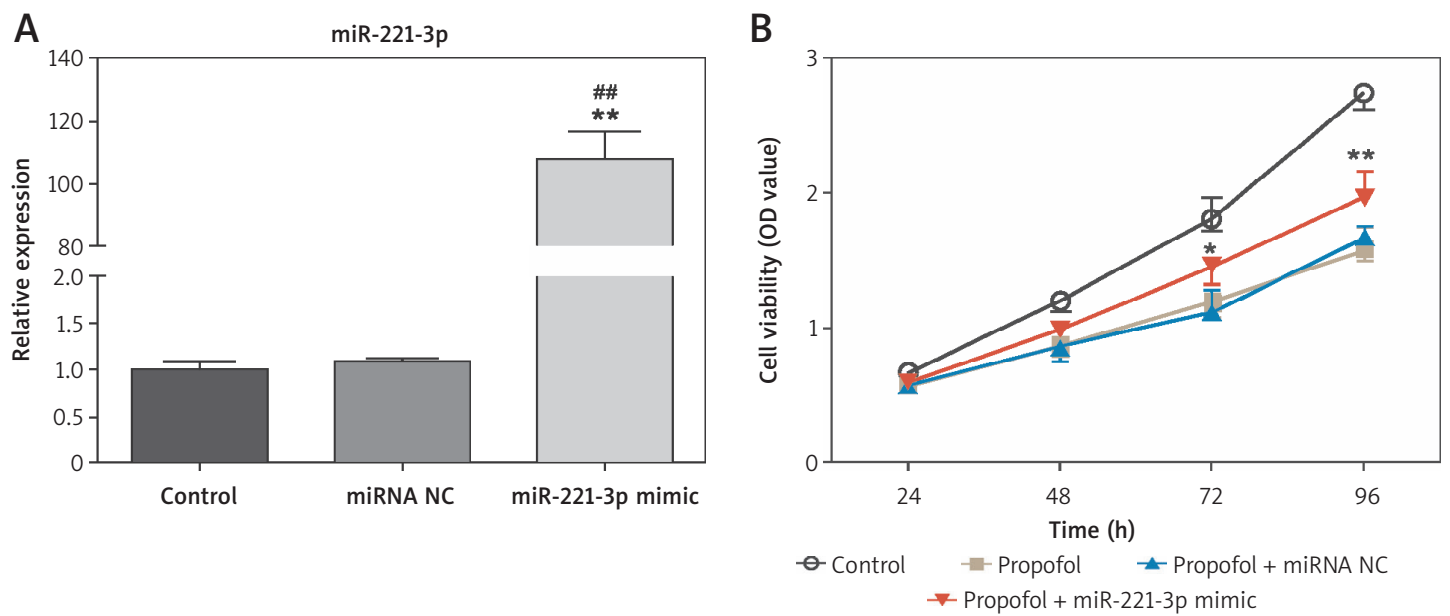

Figure 2. Overexpression of miR-221-3p attenuates cell proliferation induced by propofol treatment. A - Synthetic miR-221-3p mimic and miR-221-3p NC were transfected into purified rat astrocytes, and delivery efficiency was examined through qRT-PCR. B - Purified astrocytes divided into four groups were harvested at indicated time points for cell viability detection using CCK8. ${ }^{* *} P<0.01$ compared to the controls; ${ }^{\# \# ~} P<0.01$ compared to the miRNA NC group 
A
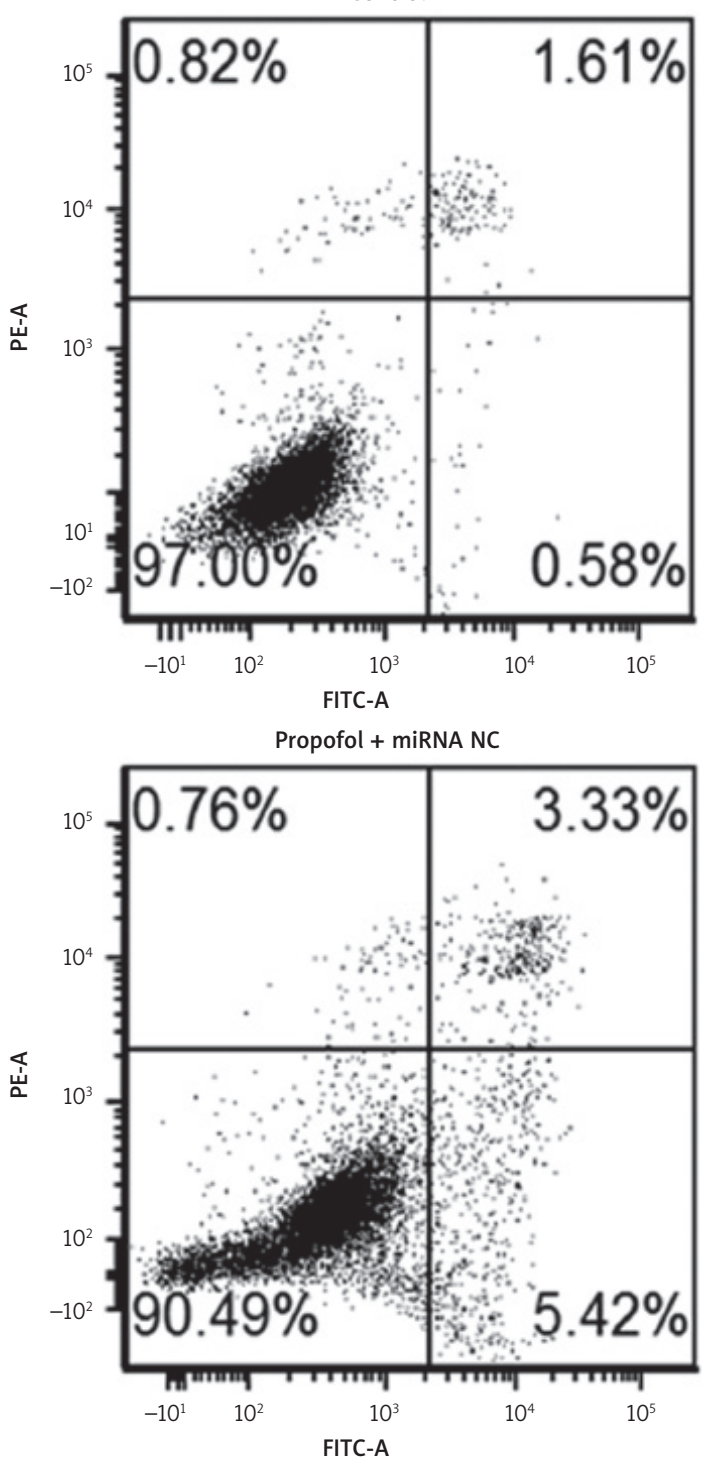

B

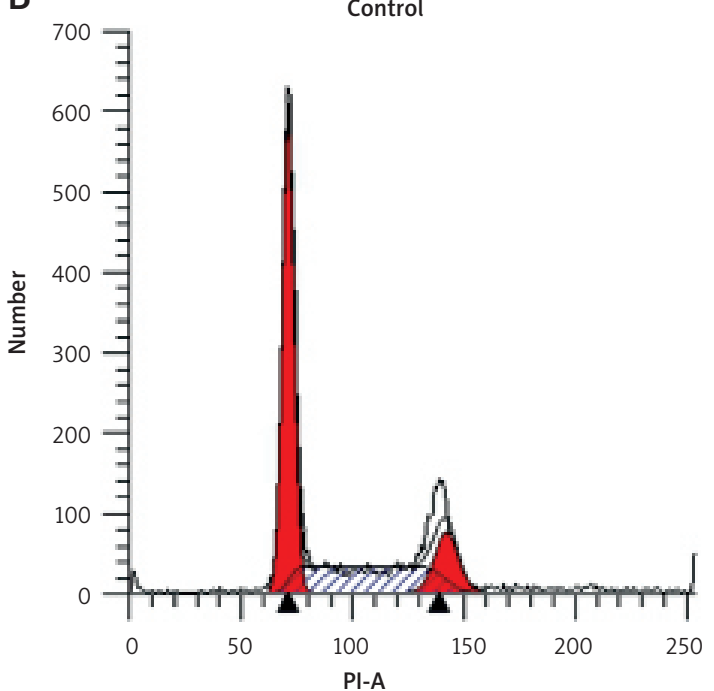

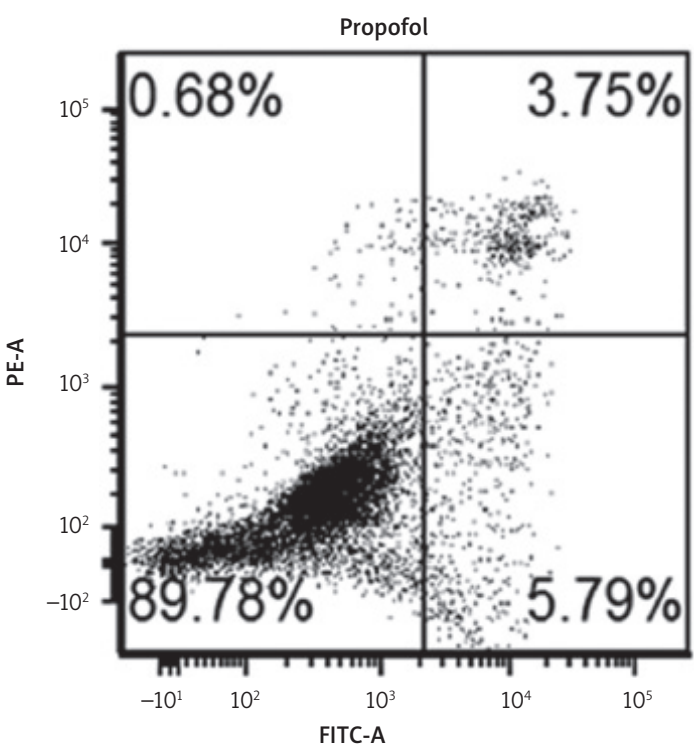
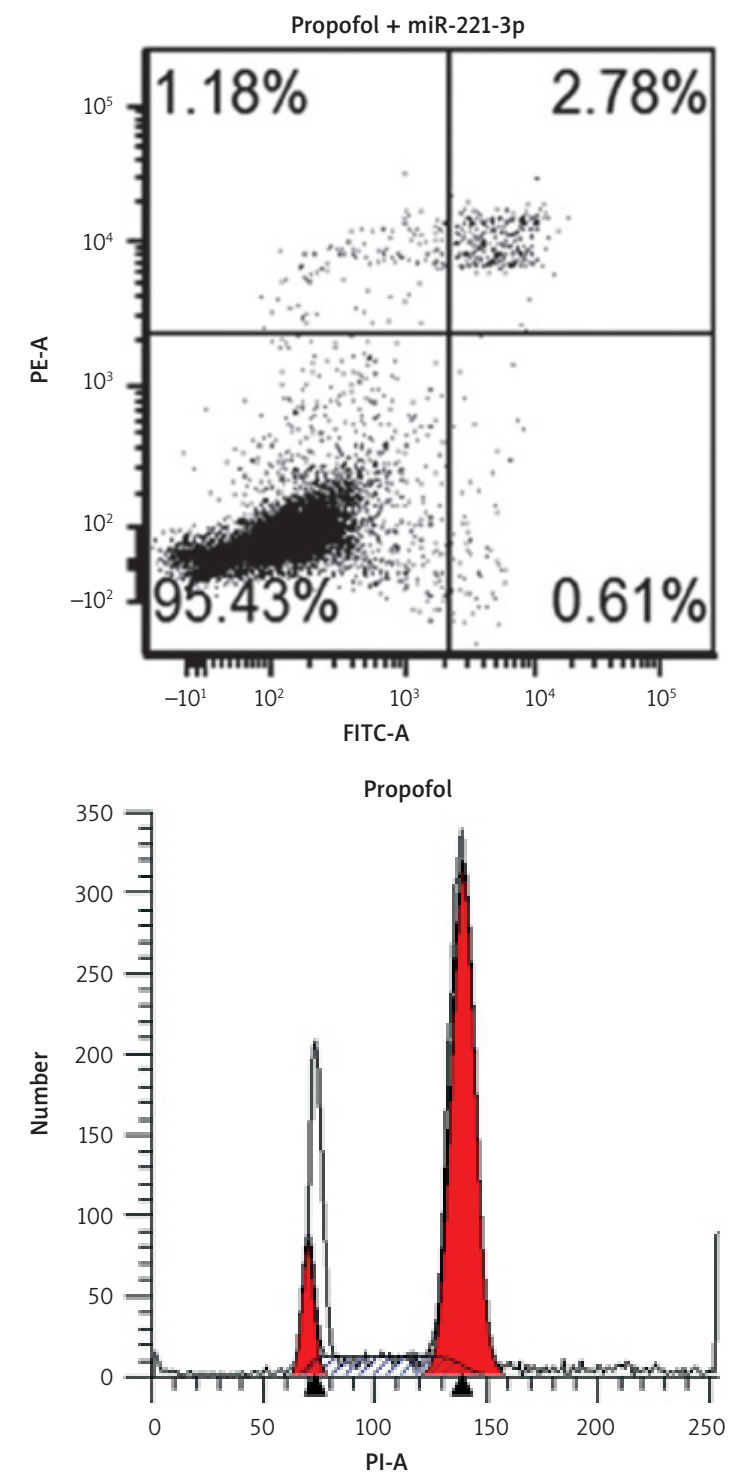

Figure 3. Overexpression of miR-221-3p modifies apoptosis and cell cycle in propofol-treated astrocytes. The blank control, NC mimic, propofol + NC mimic, and propofol + miR-221-3p mimic groups were harvested for flow cytometry assay to measure the apoptosis (A) rate and cell cycle (B) 

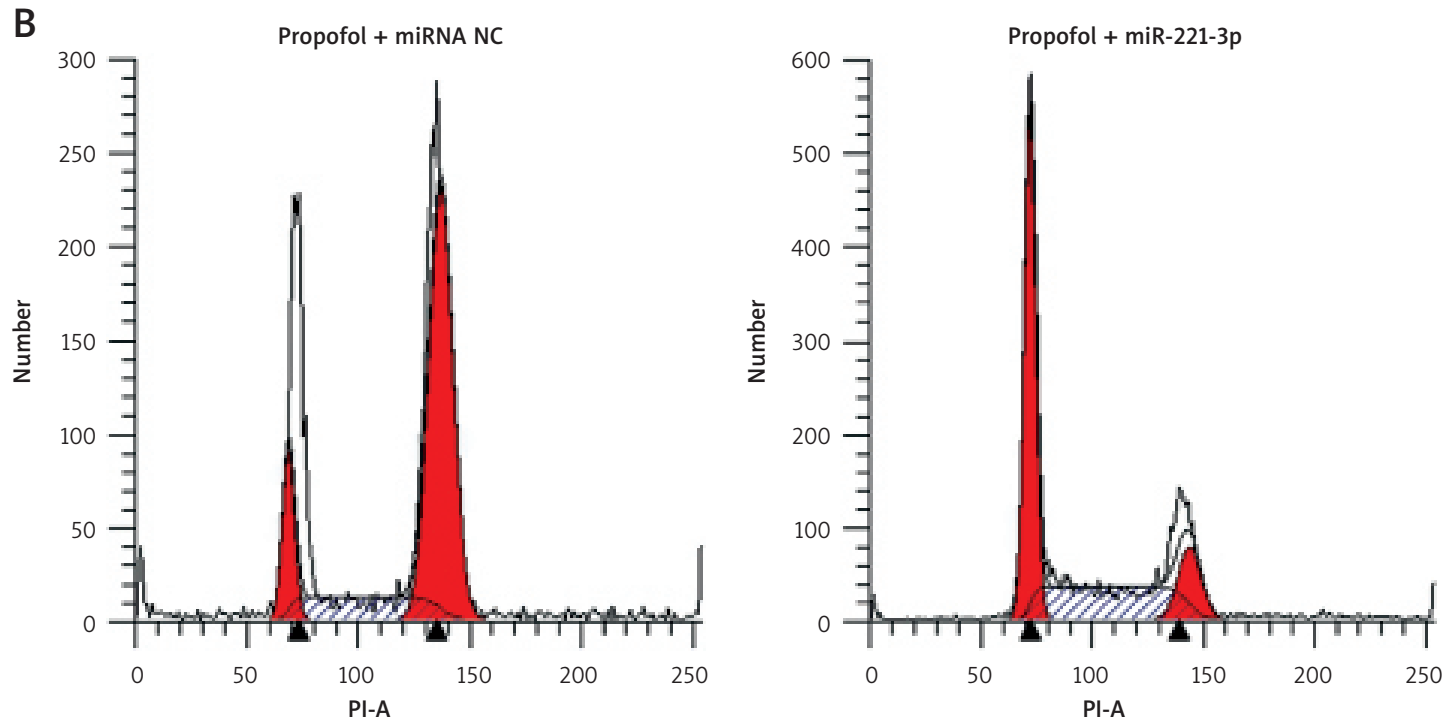

Figure 3. Cont. Rate and cell cycle (B)
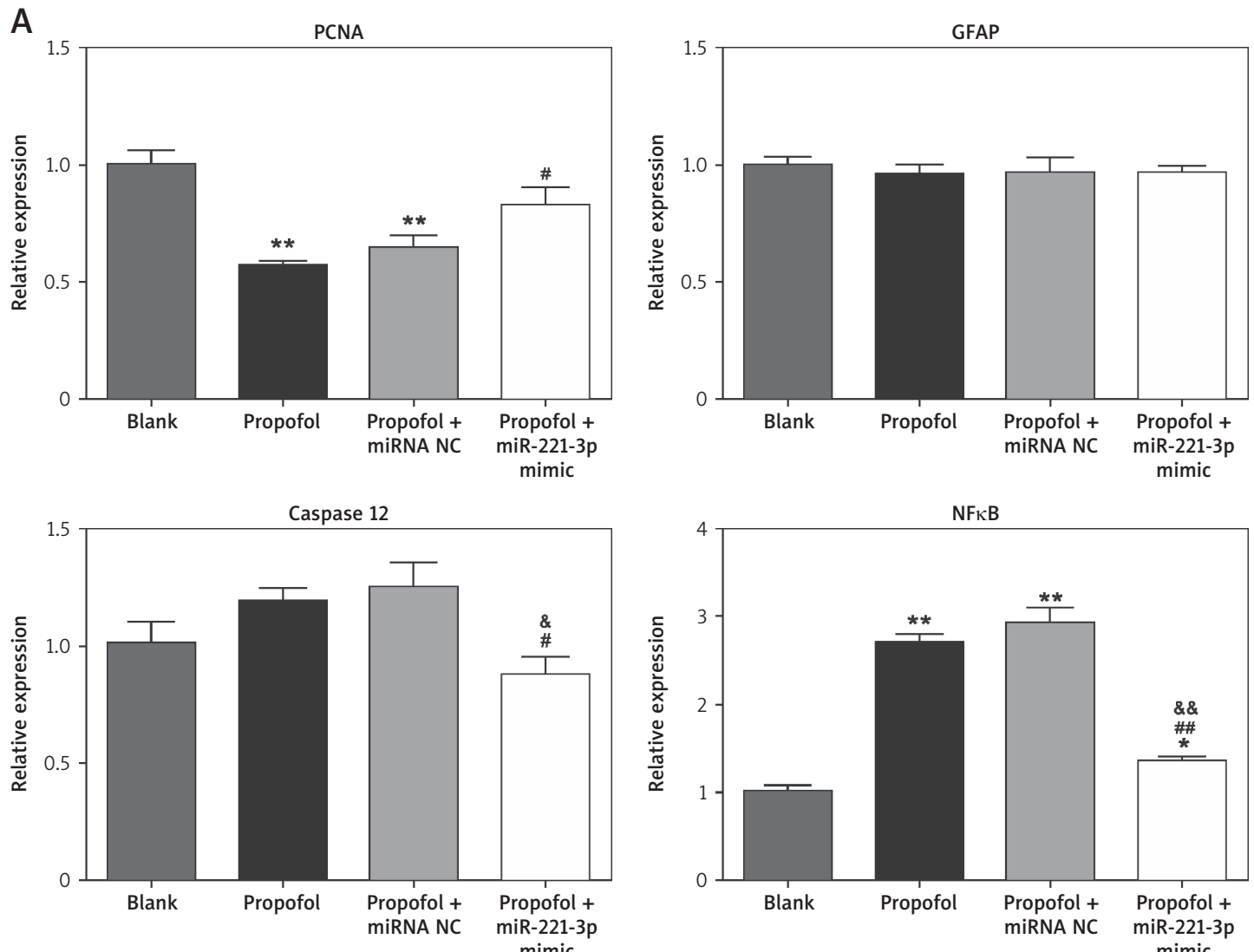

Figure 4. miR-221-3p regulates the TLR4-MyD88 signaling pathway in propofol-treated astrocytes. A - The blank control, NC mimic, propofol + NC mimic, and propofol + miR-221-3p mimic groups were harvested for qRT-PCR to quantify the mRNA levels of PCNA, GFAP, caspase-12, STAT3, GRP78, NF- $\kappa B, I L-6, I L-1 \beta$, TLR4, and MyD88. ${ }^{* *} P<0.01$ compared to the controls. ${ }^{\#} P<0.01$ compared to the miRNA NC group. ${ }^{*} P<0.05,{ }^{* *} p<0.01$ compared with the blank controls. ${ }^{\#} P<0.05,{ }^{\# \#} p<0.01$ compared with the propofol group. ${ }^{*} P<0.05$, \&\& $p<0.01$ compared to the propofol $+\mathrm{NC}$ mimic group 

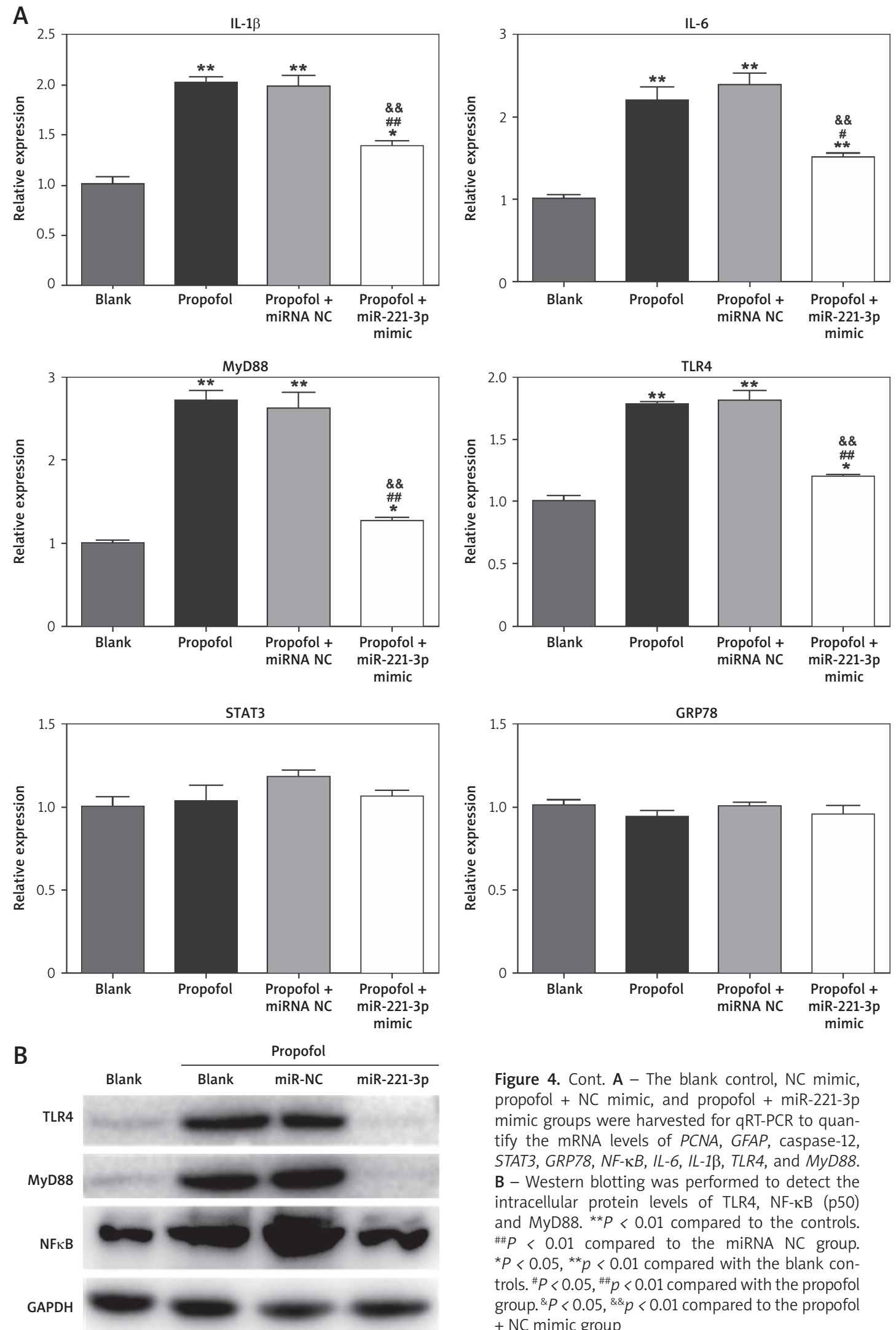

Figure 4. Cont. A - The blank control, NC mimic, propofol + NC mimic, and propofol + miR-221-3p mimic groups were harvested for qRT-PCR to quantify the mRNA levels of PCNA, GFAP, caspase-12, STAT3, GRP78, NF-KB, IL-6, IL-1B, TLR4, and MyD88. $B$ - Western blotting was performed to detect the intracellular protein levels of TLR4, NF-KB (p50) and $\mathrm{MyD} 88 .{ }^{* *} P<0.01$ compared to the controls. ${ }^{\# \# P} P<0.01$ compared to the miRNA NC group. ${ }^{*} P<0.05,{ }^{* *} p<0.01$ compared with the blank controls. $\# P<0.05, \# p<0.01$ compared with the propofol group. ${ }^{\&} P<0.05$, \&\& $p<0.01$ compared to the propofol + NC mimic group 
(STAT3), caspase-3, and glucose-regulated protein 78 (GRP78). As shown in Figure 4 A, the results indicated that PCNA mRNA level in the propofol + miR-221-3p mimic group was significantly improved when compared with the propofol group $(p<0.05)$, while there was no significant difference when compared with the propofol + miRNA NC group. In addition, the mRNA expression of GFAP, STAT3 and GRP78 in the propofol + miR221-3p mimic group was not significantly different when compared with the propofol group and propofol + miRNA NC group. The mRNA expression of caspase-12 was significantly lower when compared with the propofol group and propofol + miRNA NC group $(p<0.05)$. Moreover, the mRNA expression levels of $N F-\kappa B, M y D 88, T L R 4, I L-6$ and $I L-1 \beta$ in the propofol + miR-221-3p mimic group were markedly lower when compared with the other three groups $(p<0.01)$. When we further examined the protein expression of TRL4, MyD88 and NF- $\kappa B$, the results indicated that the overexpression of miR-221-3p dramatically decreased cellular protein levels of TLR4, MyD88 and NF- $\kappa B$ (p50) (Figure 4 B). This result indicated that the overexpression of miR-221-3p could reverse the expression of TLR4, MyD88 and NF- $\mathrm{KB}$ (p50) in astrocytes induced by propofol.

\section{TLR4 was a target of miR-221-3p}

We found that the miR-221-3p expression trend was opposite to that of TLR4 (Figure $1 \mathrm{~B}$ and Figure 4 A). Complementary sequences were observed between TLR4 3'UTR and miR-221-3p when combined with bioinformatics prediction (Figure $5 \mathrm{~A}$ ). The association between miR-221-3p and TLR4 was detected using the Dual-Luciferase Reporter Assay system. Ectopic expression of miR-221-3p significantly decreased the TLR4 3'UTR-WT luciferase activity (Figure 5 B).

\section{Knockdown of TLR4 could suppress the apoptosis rate in propofol-treated astrocytes}

To explore the function of TLR4 in propofoltreated astrocytes, TLR4 was knocked down by transfecting 293T cells that were transfected with siTLR4. The results of qRT-PCR indicated that TLR4 expression was markedly decreased after the knockdown of TLR4-1, TLR4-2, and TLR4-3 (Figure $6 \mathrm{~A}$ ). We selected TLR4-1 as the final SiRNA sequence for the following experiments. TLR4 knockdown cells markedly suppressed apoptosis compared with the cells in the siRNA controls $(p<0.01)$ (Figure $6 \mathrm{~B}$ and C). In addition, we further examined the protein related apoptosis, including bax, bcl-2, caspase 3 and caspase 9 using western blot. The results indicated that the expression
A
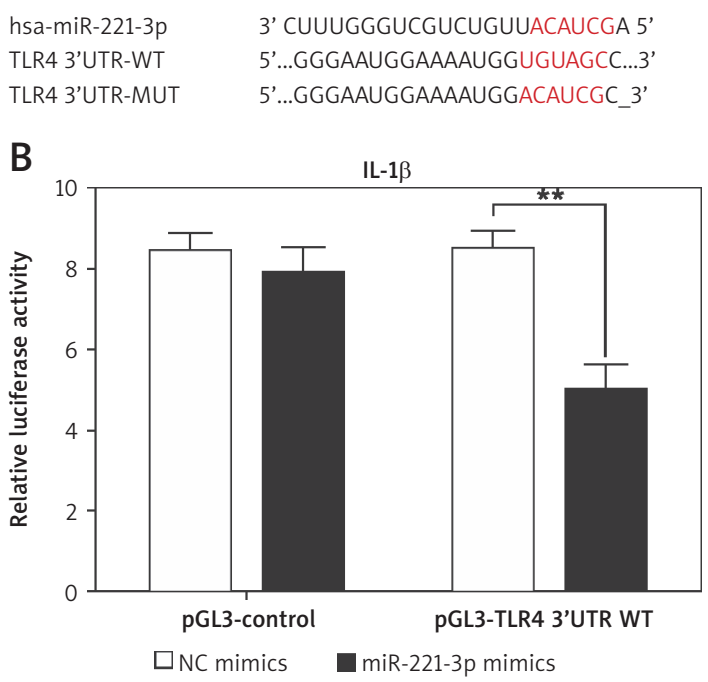

Figure 5. TLR4 is a target of miR-221-3p. Dual-Luciferase Reporter Assay was performed to identify the association between TLR4 and miR-221-3p. ${ }^{*} P<0.05$

levels of bax and caspase 3 in the propofol + siTLR4 group were lower when compared with the propofol + siNC group, while the expression levels of bcl- 2 and caspase 9 were superior when compared with the propofol + siNC group.

\section{Discussion}

In this study, we found that the miR-221-3p expression in rat astrocytes was markedly suppressed by propofol treatment. Also, the overexpression of miR-221-3p could improve the propofol-induced astrocytes viability and decrease its apoptosis and the cell number during the G2/M phase. In addition, the transfection of miR-221-3p mimics could dramatically reduce the mRNA and protein expression of MyD88, TLR4 and NF-KB in propofol-induced astrocytes. After that, TLR4 was found to be the target of miR-221-3p using the Dual-Luciferase Reporter Assay. Furthermore, knockdown of TLR4 could suppress the apoptosis rate in propofol-treated astrocytes.

Previous studies have reported that miRNA could regulate the nerve damage caused by propofol [27]. Also, Feng et al. [22] stated that miR-221-3p could be regarded as a biomarker to regulate the activation of astrocytes. However, the specific effect and potential mechanism of miR-221-3p on propofol-treated astrocytes have not yet been explored. In this study, our results indicated that miR-221-3p was an important regulator of rat astrocyte proliferation in response to propofol treatment. First, miR-221-3p expression was decreased by treatment with propofol in rat astrocytes. Furthermore, we found that miR-221-3p overexpression could improve cell growth and suppress as- 
A
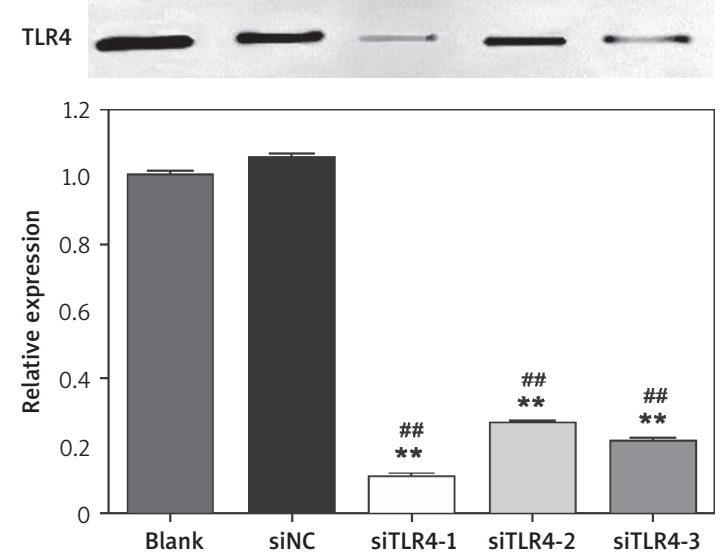

B
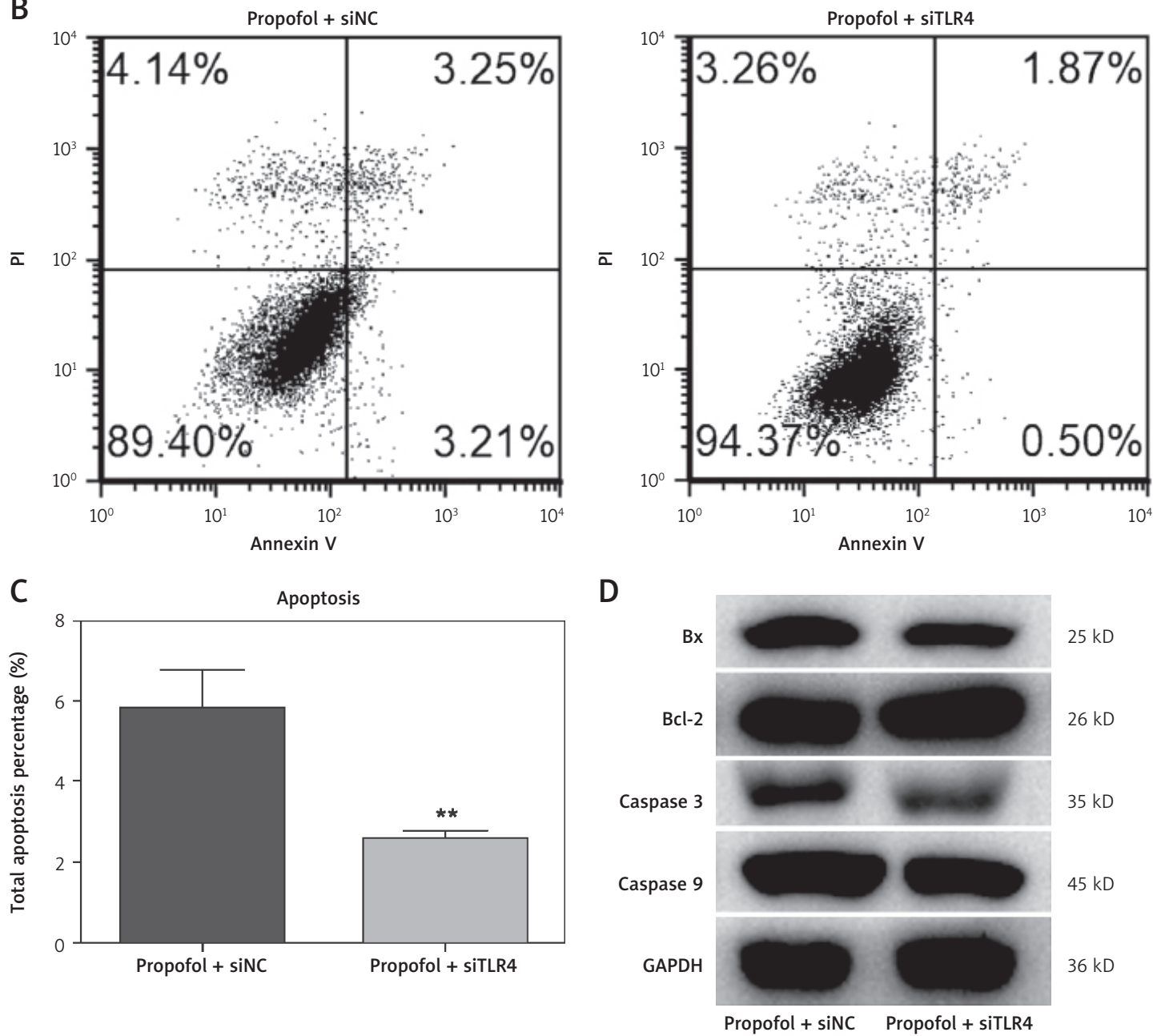

$26 \mathrm{kD}$

$35 \mathrm{kD}$

$45 \mathrm{kD}$

$36 \mathrm{kD}$

Figure 6. Knockdown of TLR4 could suppress the apoptosis rate in propofol-treated astrocytes. A - Detection of knockdown efficiency of sitlr4 by qRT-PCR. B - Knockdown of TLR4 could suppress the apoptosis rate in propofol-treated astrocytes. $\mathbf{C}$ - The apoptosis rate in astrocytes in the group of siNC with propofol treatment. D - The apoptosis rate in astrocytes in the group of siTLR4 with propofol treatment. ${ }^{* *}$ shows a highly significant difference in the blank controls $(p<0.01)$, and ${ }^{\#}$ shows a highly significant difference from the siNC group $(p<0.01)$ 
trocyte apoptosis, which were consistent with the miRNA profile data $[28,29]$.

Moreover, to probe the pathway associated with miR-221-3p, we conducted qRT-PCR detection to quantify apoptosis-related gene expression. The results indicated that the overexpression of miR-221-3p in propofol-induced astrocytes could significantly reduce the $\mathrm{mRNA}$ expression of $N F-\kappa B$,

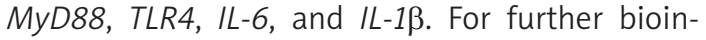
formatics analysis, we found that TLR4 was the target of miR-221-3p, which was identified using the Dual-Luciferase Reporter Assay. TLR4 is a membrane receptor that can recognize a variety of stimuli and activate related signal transduction pathways when stimulated, thereby promoting the expression of inflammatory factors [30, 31]. After knockdown of TLR4, we observed that TLR4 knockdown cells markedly suppressed the propofol-induced apoptosisrate, implyingthatmiR-221-3p affects astrocyte proliferation that was not related to ER-stress signals. Zhu et al. [17] noted that miR221-3p regulated HUVEC apoptosis and inflammation through the target of TLR4. Quero et al. [32] considered that miR-221-3p is a regulator of inflammatory M2 macrophage function induced by TLR4. The above reports suggested that miR-221-3p could mediate apoptosis and inflammation via the target of TLR4. Therefore, our studies suggested that miR-221-3p could improve the damage of CNS induced by propofol through regulating the expression of TLR4.

There are some limitations in our research. First, due to the characteristics of the multiple targets of propofol on the cell, we are currently unable to determine the way propofol enters the cell to perform its function. Therefore, our research cannot clarify the exact mechanism by which miRNA regulates the neurotoxicity caused by propofol. The TLR4-mediated NF-KB pathway need to be validated in propofol-induced astrocytes, which could provide a better understanding for the function of miRNA in propofol-induced astrocytes. In addition, in order to clarify whether propofol is toxic to the developing brain, it is necessary to conduct in vivo propofol exposure experiments in newborn animals.

\section{Conclusions}

In summary, we found that overexpression of miR-221-3p could improve the viability of propofol-induced astrocytes and decrease their apoptosis rate. In addition, the results indicated that miR-221-3p may protect astrocytes from propofol-induced damage by targeting TLR4.

\section{Conflict of interest}

The authors declare no conflict of interest.

\section{References}

1. Glen JBI. The discovery and development of propofol anesthesia: the 2018 Lasker-DeBakey Clinical Medical Research Award. JAMA 2018; 320: 1235-6.

2. Sattin D, Duran D, Visintini S, et al. Analyzing the loss and the recovery of consciousness: functional connectivity patterns and changes in heart rate variability during propofol-induced anesthesia. Front Syst Neurosci 2021; 15: 652080.

3. Bonnet MP, Mercier FJ, Vicaut E, Galand A, Keita H, Baillard C. Incidence and risk factors for maternal hypoxaemia during induction of general anaesthesia for non-elective Caesarean section: a prospective multicentre study. Br J Anaesth 2020; 125: e81-7.

4. Houben A, Ghamari S, Fischer A, Neumann C, Baehner T, Ellerkmann RK. Pediatric emergence delirium is linked to increased early postoperative negative behavior within two weeks after adenoidectomy: an observational study. Braz J Anesthesiol 2021; doi: 10.1016/ j.bjane.2021.03.008 [Online ahead of print].

5. Chidambaran V, Costandi A, D'Mello A. Correction to: Propofol: a review of its role in pediatric anesthesia and sedation. CNS Drugs 2018; 32: 873.

6. McCann ME, Soriano SG. Does general anesthesia affect neurodevelopment in infants and children? BMJ 2019; 367: 16459.

7. De Andrade LM, Isenberg SJ. Does general anesthesia or intravitreal injection affect neurodevelopment in children undergoing ophthalmic procedures? Curr Opin Ophthalmol 2019; 30: 326-30.

8. Fan Y, Zhu S, Wang J, Zhao Y, Wang X. Propofol protects against oxygen/glucose deprivation-induced cell injury via gap junction inhibition in astrocytes. Mol Med Rep 2020; 22: 2896-904.

9. Seol B, Kim YD, Cho YS. Modeling sialidosis with neural precursor cells derived from patient-derived induced pluripotent stem cells. Int J Mol Sci 2021; 22: 4386.

10. Li X, Gao Z, Xu L, Li B, Gao H. Over-expression of arginine vasopressin in magnocellular neurosecretory cells of hypothalamus and its potential relationship with development of diabetic nephropathy. Arch Med Sci 2020; 16: 1130-9.

11. Bartel DP. MicroRNAs: target recognition and regulatory functions. Cell 2009; 136: 215.

12. Bai Y, Su X, Piao L, Jin Z, Jin R. Involvement of astrocytes and microRNA dysregulation in neurodegenerative diseases: from pathogenesis to therapeutic potential. Front Mol Neurosci 2021; 14: 556215.

13. Brites D. Regulatory function of microRNAs in microglia. Glia 2020; 68: 1631-42.

14. Barbosa M, Gomes C, Sequeira C, et al. Recovery of depleted miR-146a in ALS cortical astrocytes reverts cell aberrancies and prevents paracrine pathogenicity on microglia and motor neurons. Front Cell Dev Biol 2021; 9: 634355.

15. Li L, Voloboueva L, Griffiths BB, Xu L, Giffard RG, Stary CM. MicroRNA-338 inhibition protects against focal cerebral ischemia and preserves mitochondrial function in vitro in astrocytes and neurons via COX411. Mitochondrion 2021; 59: 105-12.

16. Fukuda S, Akiyama M, Niki Y, Kawatsura R, Harada $\mathrm{H}$, Nakahama KI. Inhibitory effects of miRNAs in astrocytes on C6 glioma progression via connexin 43. Mol Cell Biochem 2021; 476: 2623-32.

17. Zhu L, Gong X, Gong J, et al. Notoginsenoside R1 upregulates miR-221-3p expression to alleviate ox-LDLinduced apoptosis, inflammation, and oxidative stress 
by inhibiting the TLR4/NF- $\mathrm{BB}$ pathway in HUVECs. Braz J Med Biol Res 2020; 53: e9346.

18. Rong J, Xu J, Liu Q, et al. Anti-inflammatory effect of up-regulated microRNA-221-3p on coronary heart disease via suppressing NLRP3/ASC/pro-caspase-1 inflammasome pathway activation. Cell Cycle 2020; 19: 147891.

19. Ye T, Zhong L, Ye X, Liu J, Li L, Yi H. miR-221-3p and miR222-3p regulate the SOCS3/STAT3 signaling pathway to downregulate the expression of NIS and reduce radiosensitivity in thyroid cancer. Exp Ther Med 2021; 21: 652.

20. Ni L, Xu J, Zhao F, et al. MiR-221-3p-mediated downregulation of MDM2 reverses the paclitaxel resistance of non-small cell lung cancer in vitro and in vivo. Eur J Pharmacol 2021; 899: 174054.

21. Pan X, Hong X, Li S, Meng P, Xiao F. METTL3 promotes adriamycin resistance in MCF-7 breast cancer cells by accelerating pri-microRNA-221-3p maturation in a m6Adependent manner. Exp Mol Med 2021; 53: 91-102.

22. Feng J, Wang M, Li M, et al. Serum miR-221-3p as a new potential biomarker for depressed mood in perioperative patients. Brain Res 2019; 1720: 146296.

23. Jiang Q, Stone CR, Elkin K, Geng X, Ding Y. Immunosuppression and neuroinflammation in stroke pathobiology. Exp Neurobiol 2021; 30: 101-12.

24. Leavy O. Innate immunity: SHP regulates TLR signalling. Nat Rev Immunol 2011; 11: 502.

25. Valentino KL, Jones EG, Kane SA. Expression of GFAP immunoreactivity during development of long fiber tracts in the rat CNS. Brain Res 1983; 9: 317-36.

26. Bignami A, Dahl D. Specificity of the glial fibrillary acidic protein for astroglia. J Histochem Cytochem 1977; 25: 466-9.

27. Guerrero Orriach JL, Escalona Belmonte JJ, Ramirez Aliaga $M$, et al. NGS of microRNAs involved in cardioprotection induced by sevoflurane compared to propofol in myocardial revascularization surgery: the ACDHUVV-16 Clinical Trial. Curr Med Chem 2021; 28: 4074-86.

28. Sun W, Pei L. microRNA expression profiling of propofoltreated developing rat hippocampal astrocytes. DNA Cell Biol 2015; 34: 511-23.

29. Creeley C, Dikranian K, Dissen G, Martin L, Olney J, Brambrink A. Propofol-induced apoptosis of neurones and oligodendrocytes in fetal and neonatal rhesus macaque brain. Br J Anaesth 2013; 110 (Suppl 1): 29-38.

30. Wang J, Lin D, Peng H, Shao J, Gu J. Cancer-derived immunoglobulin $\mathrm{G}$ promotes LPS-induced proinflammatory cytokine production via binding to TLR4 in cervical cancer cells. Oncotarget 2014; 5: 9727-43.

31. Zhang CY, Du J, Zhang R, Jin J, Qiao LY. Erythropoietin attenuates propofol-induced hippocampal neuronal cell injury in developing rats by inhibiting toll-like receptor 4 expression. Neurosci Lett 2020; 716: 134647.

32. Quero L, Tiaden AN, Hanser E, et al. miR-221-3p drives the shift of M2-macrophages to a pro-inflammatory function by suppressing JAK3/STAT3 activation. Front Immunol 2019; 10: 3087.

33. Fan C, Qiao Y, Tang M. Notoginsenoside R1 attenuates high glucose-induced endothelial damage in rat retina capillary endothelial cells by modulating the intracellular redox state. Drug Des Devel Ther 2017; 11: 3343-54.

34. Xu N, Yu K, Yu H, et al. Recombinant ricin toxin binding subunit B (RTB) stimulates production of TNF- $\alpha$ by mouse macrophages through activation of TLR4 signaling pathway. Front Pharmacol 2020; 11: 526129. 